PROCEEDINGS OF THE

AMERICAN MATHEMATICAL SOCIETY

Volume 140, Number 2, February 2012, Pages 561-574

S 0002-9939(2011)10918-3

Article electronically published on June 7, 2011

\title{
ON VARIOUS TEICHMÜLLER SPACES OF A SURFACE OF INFINITE TOPOLOGICAL TYPE
}

\author{
DANIELE ALESSANDRINI, LIXIN LIU, ATHANASE PAPADOPOULOS, AND WEIXU SU
}

(Communicated by Alexander N. Dranishnikov)

\begin{abstract}
We investigate various Teichmüller spaces associated to a surface of infinite topological type. We show that the length spectrum metric is complete. We give results and examples that compare the length spectrum Teichmüller space with the quasiconformal and the Fenchel-Nielsen Teichmüller spaces.
\end{abstract}

\section{INTRODUCTION}

In this paper, we investigate various Teichmüller spaces associated to a surface of infinite topological type, continuing works that were done in [10 and 2]. An initial impulse to these works was given in a paper by H. Shiga [14.

Let $S$ be an orientable connected surface of infinite topological type. More precisely, we assume that $S$ is obtained by gluing along their boundary components a countably infinite number of generalized pairs of pants. Here, a generalized pair of pants is a sphere with three holes, a hole being either a point removed (leaving a puncture of the pair of pants) or an open disk removed (leaving a boundary component of the pair of pants).

We study Teichmüller spaces of $S$. We recall that unlike the case of surfaces of finite type, there are several Teichmüller spaces associated to the surface $S$, each of which depends heavily set-theoretically on the choice of a basepoint for that space. Furthermore, even if we fix a basepoint, the Teichmüller space depends (again, set-theoretically) on a distance function that we put on that space. There are various distances that one can use here. For instance, one can measure distances between (equivalence classes of) hyperbolic structures by taking suprema of ratios of geodesic lengths of simple closed curves with respect to these two structures, or by taking best quasiconformal homeomorphism constants between them, or by taking best bi-Lipschitz homeomorphism constants, or the sup norm associated to Fenchel-Nielsen coordinates, and so on. We refer to the papers [10] and [2] for a discussion of such ideas. The Teichmüller spaces we obtain consequently have different names, and in this paper, we shall deal with the so-called "length-spectrum

Received by the editors August 23, 2010 and, in revised form, November 22, 2010.

2000 Mathematics Subject Classification. Primary 32G15, 30F30, 30F60.

Key words and phrases. Surfaces of infinite topological type, Teichmüller space, Teichmüller metric, quasiconformal metric, length spectrum metric, Fenchel-Nielsen coordinates, FenchelNielsen metric.

The second and fourth authors were partially supported by NSFC grants 10871211 and 11011130207.

(C)2011 American Mathematical Society 561

Reverts to public domain 28 years from publication 
Teichmüller space", "quasiconformal Teichmüller space" and "Fenchel-Nielsen Teichmüller space". Furthermore, even in the cases where these Teichmüller spaces coincide setwise, the topologies on these spaces induced by the various metrics may not be the same.

For the purpose of stating the results, let us briefly review the definitions.

We shall often use the same letter for a hyperbolic structure on a surface and for the homotopy class of this structure.

The length-spectrum Teichmüller space, $\mathcal{T}_{l s}\left(H_{0}\right)$, with basepoint a (homotopy class of hyperbolic structure $H_{0}$ on $S$, is the space of homotopy classes of hyperbolic structures $H$ on $S$ such that the ratios of lengths of simple closed geodesics measured in the metrics $H_{0}$ and $H$ are uniformly bounded (see more precisely Definition 2.1 below). This space is equipped with a natural distance $d_{l s}$ called the length-spectrum distance, obtained by taking the logarithm of the supremum of ratios of geodesic lengths between (homotopy classes of) hyperbolic structures; see formula (2.2) below.

The quasiconformal Teichmüller space, $\mathcal{T}_{q c}\left(H_{0}\right)$, with basepoint a (homotopy class of) conformal structure $H_{0}$ on $S$, is the space of homotopy classes of conformal structures $H$ on $S$ such that there exists a quasiconformal mapping homotopic to the identity between the structures $H_{0}$ and $H$ (see more precisely Definition 2.2 below). We denote this space by $\mathcal{T}_{q c}\left(H_{0}\right)$. This space is equipped with a natural distance $d_{q c}$, the quasiconformal or Teichmüller distance, given by the logarithm of the dilatation of the best quasiconformal map homotopic to the identity between the two structures; see formula (2.3) below.

A simple closed curve on $S$ is said to be essential if it is not homotopic to a point or to a puncture (but it can be homotopic to a boundary component). We let $\mathcal{S}=\mathcal{S}(S)$ be the set of isotopy classes of essential simple closed curves on $S$. Given an element $\alpha$ of $\mathcal{S}$ and a (homotopy class of) hyperbolic structure $H$ on $S$, we denote by $l_{H}(\alpha)$ the length of the unique closed $H$-geodesic in the class $\alpha$.

By an abuse of notation, we shall often identify a hyperbolic structure (respectively conformal structure) on $S$ with the homotopy class of that metric (respectively conformal structure) as an element of Teichmüller space.

A basic result that is used in comparing the two Teichmüller spaces $\left(\mathcal{T}_{l s}, d_{l s}\right)$ and $\left(\mathcal{T}_{q c}, d_{q c}\right)$ is a theorem of Wolpert stating that if $H$ and $H^{\prime}$ are two hyperbolic structures on the surface $S$, then, for any $K$-quasiconformal map $f:(S, H) \rightarrow$ $\left(S, H^{\prime}\right)$ and for any element $\alpha$ in $\mathcal{S}(S)$, we have the following inequality:

$$
\frac{1}{K} \leq \frac{l_{H^{\prime}}(f(\alpha))}{l_{H}(\alpha)} \leq K
$$

For a proof, see [1]. We refer to this result as Wolpert's inequality.

From this inequality, we obtain a natural inclusion map

$$
\mathcal{T}_{q c}\left(H_{0}\right) \hookrightarrow \mathcal{T}_{l s}\left(H_{0}\right) .
$$

In general, this inclusion map is not surjective (see [10] for an example), but it is continuous (and Lipschitz), since Wolpert's inequality also implies that for any two elements $H$ and $H^{\prime}$ in $\mathcal{T}_{q c}\left(S_{0}\right)$, we have

$$
d_{l s}\left(H, H^{\prime}\right) \leq d_{q c}\left(H, H^{\prime}\right) \text {. }
$$

We shall also use Fenchel-Nielsen coordinates for hyperbolic structures. These coordinates are defined relative to a pair of pants decomposition. The notion of 
hyperbolic pair of pants decomposition of our infinite type surface $S$ has to be used with some special care, one reason being that unlike the case of surfaces of finite type, if we are given a topological pair of pants decomposition $\mathcal{P}=\left\{C_{i}\right\}_{i \in I}$ of $S$ (where $I$ is a countably infinite set) and a hyperbolic structure $H_{0}$ on $S$, and if we replace each simple closed curve $C_{i}$ by the $H_{0}$-geodesic in its homotopy class, then some of the closed geodesics homotopic to the curves $C_{i}$ might accumulate on a geodesic of infinite length (homeomorphic to $\mathbb{R}$ ), and then the union of the closed geodesics might not be a geodesic pair of pants decomposition. Such a phenomenon can be seen in examples given by Basmajian in his paper [3. In the paper 2, we gave a necessary and sufficient condition (which we called Nielsenconvexity) under which given a hyperbolic structure on a surface of infinite type, a topological pair of pants decomposition (or, equivalently, any topological pair of pants decomposition) can be made geodesic. Consequently, when we talk about Fenchel-Nielsen coordinates for a hyperbolic surface, we shall tacitly assume that the underlying hyperbolic structure is Nielsen-convex.

In the paper [2, we also introduced the notion of a Fenchel-Nielsen Teichmüller space, $\mathcal{T}_{F N}\left(H_{0}\right)$, based at a (homotopy class of) hyperbolic structure $H_{0}$, with its associated Fenchel-Nielsen metric $d_{F N}$, relative to a fixed geodesic pair of pants decomposition $\mathcal{P}$ of $H_{0}$.

Given a pair of pants decomposition $\mathcal{P}=\left\{C_{i}\right\}_{i=\in I}$ of the surface $S$, the following condition on a hyperbolic structure $H$ was formulated by Shiga in his paper [14]:

$$
\exists M>0, \forall i \in I, \frac{1}{M} \leq l_{H}\left(C_{i}\right) \leq M .
$$

We shall say that such a hyperbolic structure $H$ satisfies Shiga's condition with respect to the pair of pants decomposition $\mathcal{P}=\left\{C_{i}\right\}, i \in I$.

In 10] (Theorem 4.14), we proved that if the base hyperbolic metric $H_{0}$ satisfies Shiga's Condition, then we have $\mathcal{T}_{q c}\left(H_{0}\right)=\mathcal{T}_{l s}\left(H_{0}\right)$ (set-theoretically).

We note that if the base topological surface is of finite type, then it is known that the length-spectrum and the quasiconformal Teichmüller spaces coincide setwise and that the topologies defined on that set by the length-spectrum and the quasiconformal metrics are the same. We can deduce this from the fact that the Teichmüller space topology is induced from the embedding of that space in the space $\mathbb{R}_{+}^{\mathcal{S}}$ of positive functions on $\mathcal{S}$, equipped with the weak topology via the length functions. That the topology induced by the length-spectrum metric coincides with this topology follows from the fact that the geodesic length functions of some finite number of elements of $\mathcal{S}$ are sufficient to parametrize Teichmüller space and to define its topology; see [6]. See also [7] and [8], where these metrics are discussed.

The case of surfaces of infinite type is different. The first negative result in this direction is a result by Shiga, who proved in [14] (Theorem 1.1) that there exists a hyperbolic structure $H_{0}$ on a surface of infinite type and a sequence $\left(H_{n}\right), n \geq 1$, of hyperbolic structures in $\mathcal{T}_{l s}\left(H_{0}\right)$ which (when they are regarded as conformal structures) are at the same time in $\mathcal{T}_{q c}\left(H_{0}\right)$ and satisfy

$$
d_{l s}\left(H_{n}, H_{0}\right) \rightarrow 0, \text { while } d_{q c}\left(H_{n}, H_{0}\right) \rightarrow \infty .
$$

This shows that the metrics $d_{l s}$ and $d_{q c}$ do not induce the same topology on $\mathcal{T}_{q c}\left(H_{0}\right)$.

In the same paper, Shiga showed that if the hyperbolic metric $H_{0}$ satisfies property (1.4), then $d_{l s}$ and $d_{q c}$ induce the same topology on $\mathcal{T}_{q c}(S)$. 
Furthermore, Shiga showed that there exists a Riemann surface $S_{0}$ of infinite type such that the length spectrum distance $d_{l s}$ restricted to the quasiconformal Teichmüller space $\mathcal{T}_{q c}\left(S_{0}\right)$ is not complete [14, Corollary 1.1]. We shall give below (Example 5.1) another example of this phenomenon by a construction that is probably simpler than the one of Shiga. The hyperbolic structure in this example is also different from the one given by Shiga, because in our example the surface (as a metric space) is complete, whereas in Shiga's example it is not.

We prove below (Proposition 3.2) that for some base hyperbolic structures $H_{0}$, we have $\mathcal{T}_{l s}\left(H_{0}\right) \not \subset \mathcal{T}_{F N}\left(H_{0}\right)$. We also give an example of a hyperbolic structure $H_{0}$ and a sequence of points $x_{n}, n=1, \ldots$, in $\mathcal{T}_{l s}\left(H_{0}\right) \cap \mathcal{T}_{F N}\left(H_{0}\right)$ such that $\lim _{n \rightarrow \infty} d_{l s}\left(x_{n}, H_{0}\right)=0$, while $\lim _{n \rightarrow \infty} d_{F N}\left(x_{n}, H_{0}\right)=\infty$ (Proposition 3.3).

The length spectrum metric on any Teichmüller space of a conformally finite type Riemann surface is complete (see [10, Theorem 2.25]). The proof given in [10] does not extend to the case of Teichmüller spaces of surfaces of infinite topological type. We prove this result for surfaces of infinite topological type in $₫$ below. More precisely, we prove that for any base hyperbolic metrics $H_{0}$ on $S$, the metric space $\left(\mathcal{T}_{l s}\left(H_{0}\right), d_{l s}\right)$ is complete (Theorem 4.5). This result answers a question raised in [10] (Question 2.22).

\section{The LENGTH SPECTRUM AND THE QUASICONFORMAL TEICHMÜLLER SPACES}

For the reader's convenience, we briefly review a few basic facts about the length spectrum and the quasiconformal Teichmüller spaces.

All the homotopies of a surface that we consider in this paper preserve the punctures and preserve setwise the boundary components at all times.

Throughout this section, $H_{0}$ is a fixed hyperbolic structure on the surface $S$, called the base hyperbolic structure. Given a hyperbolic structure $H$ on $S$ and a homeomorphism $f:\left(S, H_{0}\right) \rightarrow(S, H)$, we define the length-spectrum constant of $f$ to be the quantity

$$
L(f)=\sup _{\alpha \in \mathcal{S}(H)}\left\{\frac{l_{H^{\prime}}(f(\alpha))}{l_{H}(\alpha)}, \frac{l_{H}(\alpha)}{l_{H^{\prime}}(f(\alpha))}\right\} .
$$

This quantity depends only on the homotopy class of $f$. We say that $f$ is lengthspectrum bounded if $L(f)<\infty$.

In the setting of the length spectrum Teichmüller space, we consider the collection of hyperbolic structures $H$ on $S$ such that the identity map Id : $\left(S, H_{0}\right) \rightarrow$ $(S, H)$ is length-spectrum bounded. Given two such hyperbolic structures $H$ and $H^{\prime}$, we write $H \sim H^{\prime}$ if there exists an isometry (or, equivalently, a length spectrum preserving homeomorphism) from $(S, H)$ to $\left(S, H^{\prime}\right)$ which is homotopic to the identity. The relation $\sim$ is an equivalence relation on the set of length-spectrum bounded hyperbolic structures $H$ with respect to the basepoint $H_{0}$.

Definition 2.1. The length-spectrum Teichmüller space $\mathcal{T}_{l s}\left(H_{0}\right)$ is the space of $\sim$-equivalence classes of length-spectrum bounded hyperbolic structures. The basepoint of this Teichmüller space is the equivalence class $H_{0}$.

We note that the fact that we do not ask our homotopies to preserve pointwise the boundary of the surface corresponds to working with what is usually called the reduced Teichmüller space as opposed to the non-reduced Teichmüller space. In the latter case, the homotopies that define the equivalence relation are required to 
induce the identity map on each boundary component. The basic example of the non-reduced theory is the theory of the Teichmüller space of the unit disc, which is infinite-dimensional. Since all the Teichmüller spaces that we consider in this paper are reduced, we shall use, for simplicity, the terminology Teichmüller space instead of reduced Teichmüller space.

The topology of $\mathcal{T}_{l s}\left(H_{0}\right)$ is induced by the length-spectrum metric $d_{l s}$, defined by taking the distance $d_{l s}\left(H, H^{\prime}\right)$ between two points in $\mathcal{T}_{l s}\left(H_{0}\right)$ represented by two marked hyperbolic surfaces $(f, H)$ and $\left(f^{\prime} H^{\prime}\right)$ to be

$$
d_{l s}\left(H, H^{\prime}\right)=\frac{1}{2} \log L\left(f^{\prime} \circ f^{-1}\right) .
$$

(It may be useful to recall here that the length-spectrum constant of a lengthspectrum bounded homeomorphism only depends on the homotopy class of such a homeomorphism.)

The fact that the function $d_{l s}$ satisfies the properties of a metric is straightforward, except perhaps for the axiom saying that if $d_{l s}\left(H, H^{\prime}\right)=0$ for $H, H^{\prime} \in$ $\mathcal{T}_{l s}\left(H_{0}\right)$, then $H=H^{\prime}$; see [10].

A Riemann surface is a one-dimensional complex manifold. Riemann surface structures are also called conformal structures.

Given a real number $K \geq 1$, a homeomorphism $f: R \rightarrow R^{\prime}$ between two Riemann surfaces is said to be $K$-quasiconformal if $f$ has locally integrable distributional derivatives satisfying at each point the inequality

$$
\left|f_{\bar{z}}\right| \leq \frac{K-1}{K+1}\left|f_{z}\right| .
$$

The quasiconformal dilatation, or, for short, the dilatation of $f$, is the infimum of the real numbers $K$ for which $f$ is $K$-quasiconformal.

In the setting of the quasiconformal Teichmüller space with basepoint a conformal structure $R_{0}$ on $S$, we consider only conformal structures $R$ on $S$ such that the identity map Id $:\left(S, R_{0}\right) \rightarrow(S, R)$ is quasiconformal. Given two such conformal structures $R$ and $R^{\prime}$, we write $R \sim R^{\prime}$ if there exists a conformal map from $(S, R)$ to $\left(S^{\prime}, R^{\prime}\right)$ which is homotopic to the identity. The relation $\sim$ is an equivalence relation on the set of conformal structures $R$ on $S$ with respect to the basepoint $R_{0}$.

Definition 2.2. Consider a conformal structure $R_{0}$ on $X$. Its quasiconformal Teichmüller space, $\mathcal{T}_{q c}\left(R_{0}\right)$, is the set of $\sim$-equivalence classes of conformal structures on $S$.

The space $\mathcal{T}_{q c}\left(R_{0}\right)$ is equipped with the quasiconformal metric, also called the Teichmüller metric, of which we also recall the definition: Given two (equivalence classes of) conformal structures $R$ and $R^{\prime}$ on $S$, their quasiconformal distance $d_{q c}\left(R, R^{\prime}\right)$ is defined as

$$
d_{q c}\left(R, R^{\prime}\right)=\frac{1}{2} \log \inf \{K(f)\}
$$

where the infimum is taken over quasiconformal dilatations $K(f)$ of homeomorphisms $f:(S, R) \rightarrow\left(S, R^{\prime}\right)$ which are homotopic to the identity.

The equivalence class of the marked Riemann surface $R_{0}$ is the basepoint of $\mathcal{T}_{q c}\left(R_{0}\right)$. 
We refer to Nag [1] for an exposition of the quasiconformal theory of infinitedimensional Teichmüller spaces. In particular, it is known that the quasiconformal metric is complete.

Douady and Earle gave in [5] a proof of the fact that any quasiconformal Teichmüller space $\mathcal{T}_{q c}\left(R_{0}\right)$ is contractible (see [5, Theorem 3], where this result is also attributed to Tukia). It is unknown whether the length spectrum Teichmüller spaces are contractible.

\section{The Fenchel-Nielsen Teichmüller spaces}

We shall consider Fenchel-Nielsen coordinates for spaces of homotopy classes of hyperbolic structures on $S$. We carried out in [2] a study of these parameters in the setting of surfaces of infinite type. These parameters are associated to a fixed geodesic pair of pants decomposition $\mathcal{P}=\left\{C_{i}\right\}_{i \in I}$ on a hyperbolic surface. The boundary components of the surface (if they exist) are all homeomorphic to circles and are in $\mathcal{P}$. Fenchel-Nielsen coordinates are defined in the same way as the Fenchel-Nielsen parameters associated to geodesic pair of pants decompositions in the case of surfaces of finite type, but some care has to be taken regarding the existence of a geodesic pair of pants decomposition in the infinite type case. In the paper 2] we gave a necessary and sufficient condition on a hyperbolic structure on a surface of infinite type $S$ so that a topological pair of pants decomposition of $S$ can be made geodesic (see [2, Theorem 4.5]). We called this condition Nielsen-convexity.

Given a hyperbolic structure $H$ and a geodesic pair of pants decomposition on $S$, to each homotopy class of closed geodesic $C_{i} \in \mathcal{P}$ we associate a length parameter and a twist parameter. The length parameter is the familiar quantity $\left.l_{H}\left(C_{i}\right) \in\right] 0, \infty[$; that is, it is the length of the $H$-geodesic in the homotopy class $C_{i}$. The twist parameter $\theta_{H}\left(C_{i}\right)$ is defined only if $C_{i}$ is not the homotopy class of a boundary component of $S$, and it measures the relative twist amount along the geodesic in the class $C_{i}$ between the two generalized pairs of pants that have this geodesic in common (the two pairs of pants may be the same). The definition is the same as the one that is done in the case of surfaces of finite type. A precise definition of the twist parameters is contained in [15. Theorem 4.6.23]. The twist amount per unit time along the (geodesic in the class) $C_{i}$ is chosen to be proportional (and not necessarily equal) to arclength along that curve, and we follow the convention, as in [2, that a complete positive Dehn twist along the curve $C_{i}$ changes the twist parameter by addition of $2 \pi$. Thus, in some sense, the parameter $\theta_{H}\left(C_{i}\right)$ that we are using is an "angle" parameter.

The Fenchel-Nielsen parameters of $H$ make up the collection of pairs $\left(\left(l_{H}\left(C_{i}\right), \theta_{H}\left(C_{i}\right)\right)\right)_{i \in I}$, where it is understood that if $C_{i}$ is homotopic to a boundary component, then it has no associated twist parameter, and instead of a pair $\left(l_{H}\left(C_{i}\right), \theta_{H}\left(C_{i}\right)\right)$, we have a single parameter $l_{H}\left(C_{i}\right)$.

If two hyperbolic structures on $S$ are equivalent, then their Fenchel-Nielsen parameters are the same.

Given two hyperbolic metrics $H$ and $H^{\prime}$ on $S$, we define their Fenchel-Nielsen distance with respect to $\mathcal{P}$ as

$$
d_{F N}\left(H, H^{\prime}\right)=\sup _{i \in I} \max \left(\left|\log \frac{l_{H}\left(C_{i}\right)}{l_{H^{\prime}}\left(C_{i}\right)}\right|,\left|l_{H}\left(C_{i}\right) \theta_{H}\left(C_{i}\right)-l_{H^{\prime}}\left(C_{i}\right) \theta_{H^{\prime}}\left(C_{i}\right)\right|\right) \text {, }
$$


again with the convention that if $C_{i}$ is the homotopy class of a boundary component of $S$, then there is no twist parameter to be considered.

Given two hyperbolic structures $H$ and $H^{\prime}$ on $S$, we say that they are FenchelNielsen bounded (relatively to $\mathcal{P}$ ) if their Fenchel-Nielsen distance is finite. FenchelNielsen boundedness is an equivalence relation.

Let $H_{0}$ be a homotopy class of a hyperbolic structure on $S$, which we shall consider as a base element of Teichmüller space. We consider the collection of homotopy classes of hyperbolic structures $H$ which are Fenchel-Nielsen bounded from $H_{0}$ with respect to $\mathcal{P}$. Given two such homotopy classes of hyperbolic structures $H$ and $H^{\prime}$, we write $H \sim H^{\prime}$ if there exists an isometry from $(S, H)$ to $\left(S, H^{\prime}\right)$ which is homotopic to the identity. The relation $\sim$ is an equivalence relation on the set of Fenchel-Nielsen bounded homotopy classes of hyperbolic surfaces $H$ based at $H_{0}$.

Definition 3.1 (Fenchel-Nielsen Teichmüller space). The Fenchel-Nielsen Teichmüller space with respect to $\mathcal{P}$ and with basepoint $H_{0}$, denoted by $\mathcal{T}_{F N}\left(H_{0}\right)$, is the space of $\sim$-equivalence classes of hyperbolic structures which are Fenchel-Nielsen bounded relative to $H_{0}$ and $\mathcal{P}$.

The function $d_{F N}$ defined in (3.1) is clearly a distance function on $\mathcal{T}_{F N}\left(H_{0}\right)$. The basepoint of this Teichmüller space is the homotopy class $H_{0}$.

We shall call the distance $d_{F N}$ on $\mathcal{T}_{F N}\left(H_{0}\right)$ the Fenchel-Nielsen distance relative to the pair of pants decomposition $\mathcal{P}$. The map

$$
\mathcal{T}_{F N}\left(H_{0}\right) \ni H \mapsto\left(\log \left(l_{H}\left(C_{i}\right)\right)-\log \left(l_{H_{0}}\left(C_{i}\right)\right), l_{H}\left(C_{i}\right) \theta_{H}\left(C_{i}\right)\right)_{i \in I} \in \ell^{\infty}
$$

is an isometric bijection between $\mathcal{T}_{F N}\left(H_{0}\right)$ and the sequence space $l^{\infty}$. It follows from general properties of $l^{\infty}$-norms that the Fenchel-Nielsen distance on $\mathcal{T}_{F N}\left(H_{0}\right)$ is complete.

We prove in the next two propositions that we have in general $\mathcal{T}_{l s}\left(H_{0}\right) \not \subset$ $\mathcal{T}_{F N}\left(H_{0}\right)$ and that the length-spectrum distance and the Fenchel-Nielsen distance might behave quite differently.

Proposition 3.2. Let $H_{0}$ be a hyperbolic structure on $S$ and let $\mathcal{T}_{F N}\left(H_{0}\right)$ be the Fenchel-Nielsen Teichmüller space corresponding to the pants decomposition $\mathcal{P}=$ $\left\{C_{i}\right\}$. Suppose there is a subsequence $C_{i_{n}}, n=1,2, \ldots$, of $C_{i}$ in the interior of $S$ whose lengths tend to 0 . Then there exists an element $H$ in $\mathcal{T}_{l s}\left(H_{0}\right)$ with $H \notin$ $\mathcal{T}_{F N}\left(H_{0}\right)$.

Proof. To simplify notation, we denote by $C_{n}, n=1,2 \ldots$ the subsequence $C_{i_{n}}, n=$ $1,2, \ldots$ We assume that the lengths satisfy $l_{H_{0}}\left(C_{n}\right)=\epsilon_{n}$ with $\lim _{n \rightarrow \infty} \epsilon_{n}=0$.

Let

$$
t_{n}=\frac{\left[\log \left|\log \epsilon_{n}\right|\right]}{\epsilon_{n}}+1, n=1,2, \ldots,
$$

where $[r]$ denotes the integral part of the real number $r$.

For each $n=1,2, \ldots$, let $H_{n}$ be the hyperbolic metric obtained from $H_{0}$ by the $t_{n}$-th power of the positive Dehn twist about $\alpha_{n}$. We take all the positive Dehn twists to be supported on disjoint annuli, we let $T$ be the infinite composition $\tau_{1} \circ \tau_{2} \circ \ldots$, and we set $H=T\left(H_{0}\right)$. For every $n=1,2, \ldots$, we have, from the definition of the Fenchel-Nielsen distance,

$$
\begin{aligned}
\left.d_{F N}\left(H_{0}, H\right)\right) & \geq 2 \pi l_{H_{0}}\left(C_{n}\right) t_{n} \\
& \geq 2 \pi \log \left|\log \epsilon_{n}\right| .
\end{aligned}
$$


Since $\lim _{n \rightarrow \infty} \epsilon_{n}=0$, we obtain $d_{F N}\left(H_{0}, H\right)=\infty$.

The proof that $d_{l s}\left(H_{0}, H\right)<\infty$ is given in [10, Proposition 4.7].

Proposition 3.3. Let $H_{0}$ be a hyperbolic structure on $S$ and $\mathcal{T}_{F N}\left(H_{0}\right)$ be the Fenchel-Nielsen Teichmüller space corresponding to the pants decomposition $\mathcal{P}=$ $\left\{C_{i}\right\}$. Suppose that there is a sequence of homotopy classes $C_{i_{n}}, n=1,2, \ldots$ in the interior of $S$ whose lengths tend to 0 . Then there exists a sequence of elements $H_{n}, n=1,2, \ldots$, in $\mathcal{T}_{l s}\left(H_{0}\right) \cap \mathcal{T}_{F N}\left(H_{0}\right)$ such that $\lim _{n \rightarrow \infty} d_{l s}\left(H_{n}, H_{0}\right)=0$, while $\lim _{n \rightarrow \infty} d_{F N}\left(H_{n}, H_{0}\right)=\infty$.

Proof. We use the notation of the proof of Proposition 3.2 , and again we denote by $C_{n}, n=1,2 \ldots$, the subsequence $C_{i_{n}}, n=1,2, \ldots$ We take the same definitions of $\epsilon_{n}$, of $t_{n}$ and of the multiple Dehn twists $\tau_{n}$ supported on disjoint annuli, and we set $H_{n}=\tau_{n}\left(H_{0}\right)$. Then for each $n=1,2, \ldots$, we have

$$
d_{F N}\left(H_{0}, H_{n}\right) \geq 2 \pi \log \left|\log \epsilon_{n}\right| .
$$

Since the above inequality is valid for any $n \geq 1$ and since $\lim _{n \rightarrow \infty} \epsilon_{n}=0$, we have $\lim _{n \rightarrow \infty} d_{F N}\left(H_{0}, H_{n}\right)=\infty$.

Next we show that

$$
\lim _{n \rightarrow \infty} d_{l s}\left(H_{0}, H_{n}\right)=\lim _{n \rightarrow \infty} \log \sup _{\alpha \in \mathcal{S}(X)}\left\{\frac{l_{H_{n}}(\alpha)}{l_{H_{0}}(\alpha)}, \frac{l_{H_{0}}(\alpha)}{l_{H_{n}}(\alpha)}\right\}=0 .
$$

The proof is adapted from the proof of Propositions 2.13 and 4.7 of [10].

Let $\alpha$ be an arbitrary homotopy class of essential curves in $S$. For $i \in I$, if $i\left(\alpha, C_{n}\right)=0$, then $l_{H_{n}}(\alpha)=l_{H_{0}}(\alpha)$. Assume now that $i\left(\alpha, C_{n}\right) \neq 0$. By the Collar Lemma (see [4), on any hyperbolic surface $H$ any closed geodesic whose length $\epsilon$ is sufficiently small has an embedded collar neighborhood of width $|\log \epsilon|$. Thus, we can write, for all $n \geq 0$,

$$
l_{H_{0}}(\alpha) \geq i\left(\alpha, \alpha_{n}\right)\left|\log \epsilon_{n}\right| .
$$

From the definition of a Dehn twist, we have

$$
l_{H_{n}}(\alpha) \leq l_{H_{0}}(\alpha)+i\left(\alpha, C_{n}\right) l_{H_{0}}\left(C_{n}\right) t_{n} .
$$

Thus, we obtain

$$
\begin{aligned}
\frac{l_{H_{n}}(\alpha)}{l_{H_{0}}(\alpha)} & \leq 1+\frac{i\left(\alpha, C_{n}\right) \epsilon_{n} t_{n}}{l_{H_{0}}(\alpha)} \\
& \leq 1+\frac{\log \left|\log \epsilon_{n}\right|}{\left|\log \epsilon_{n}\right|}
\end{aligned}
$$

Note that $\lim _{n \rightarrow \infty} \frac{l_{H_{n}}(\alpha)}{l_{H_{0}}(\alpha)} \leq 1$. In the same way, we can prove that $\lim _{n \rightarrow \infty} \frac{l_{H_{n}}(\alpha)}{l_{H_{0}}(\alpha)}$ $\geq 1$. This gives $\lim _{n \rightarrow \infty} d_{l s}\left(H_{0}, H_{n}\right)=0$.

\section{Completeness of the Length spectrum metriC}

In this section, $H_{0}$ is a hyperbolic structure on $S,\left(\mathcal{T}_{l s}\left(H_{0}\right), d_{l s}\right)$ is the lengthspectrum Teichmüller space based at this point, equipped with the length-spectrum distance, and $\mathcal{P}=\left\{C_{i}\right\}_{i \in I}$ is a hyperbolic pair of pants decomposition of $H_{0}$. For every hyperbolic structure $H$ on $S$, we denote by $\left(l_{H}\left(C_{i}\right), \theta_{H}\left(C_{i}\right)\right)_{i \in I}$ its FenchelNielsen coordinates with respect to $\mathcal{P}$. 
Lemma 4.1. Let $\left(x_{n}\right) \subset \mathcal{T}_{l s}\left(H_{0}\right)$ be a sequence converging to a point $x$ in $\mathcal{T}_{l s}\left(H_{0}\right)$. Then for all $i \in I$ we have $l_{x_{n}}\left(C_{i}\right) \rightarrow l_{x}\left(C_{i}\right)$ and $\theta_{x_{n}}\left(C_{i}\right) \rightarrow \theta_{x}\left(C_{i}\right)$.

Proof. From the definition of the length-spectrum distance, for every simple closed curve $\gamma \subset S$ we have $l_{x_{n}}(\gamma) \rightarrow l_{x}(\gamma)$. In particular, $l_{x_{n}}\left(C_{i}\right) \rightarrow l_{x}\left(C_{i}\right)$. For every curve $C_{i}$, we consider an essential simple closed curve $\beta_{i}$ which is not homotopic to it, which intersects it in a minimal number of points (this number is 1 or 2) and which is disjoint from $C_{j}$ for any $j \neq i$. We let $\beta_{i}^{\prime}$ be the image of $\beta_{i}$ by the Dehn twist along $C_{i}$. We also have $l_{x_{n}}\left(\beta_{i}\right) \rightarrow l_{x}\left(\beta_{i}\right)$. By the formulae in [13, the absolute value of the twist parameter along $C_{i}$ is a continuous function of the length of the curves $C_{i}, \beta_{i}$ and of the other curves $C_{j}$ at the boundaries of the pair of pants containing $C_{i}$. Hence $\left|\theta_{x_{n}}\left(C_{i}\right)\right| \rightarrow\left|\theta_{x}\left(C_{i}\right)\right|$. If $\theta_{x}\left(C_{i}\right)=0$ we are done; otherwise note that by the same argument, using $\beta_{i}^{\prime}$ instead of $\beta_{i}$, we have $\left|\theta_{x_{n}}\left(C_{i}\right)+2 \pi\right| \rightarrow\left|\theta_{x}\left(C_{i}\right)+2 \pi\right|$. Hence for $n$ large enough, $\theta_{x_{n}}\left(C_{i}\right)$ and $\theta_{x}\left(C_{i}\right)$ have the same sign.

One may ask whether the converse of this lemma is true: i.e. if $\left(x_{n}\right) \subset \mathcal{T}_{l s}\left(H_{0}\right)$ is some sequence and if $x \in \mathcal{T}_{l s}\left(H_{0}\right)$ is such that $l_{x_{n}}\left(C_{i}\right) \rightarrow l_{x}\left(C_{i}\right)$ and $\theta_{x_{n}}\left(C_{i}\right) \rightarrow$ $\theta_{x}\left(C_{i}\right)$, then is it true that $\left(x_{n}\right) \rightarrow x$ in the length-spectrum metric?

We prove a result of this kind under an additional hypothesis on $\left(x_{n}\right)$; see Lemma 4.4

Lemma 4.2. Let $\left(x_{n}\right) \subset \mathcal{T}_{l s}\left(H_{0}\right)$ be a Cauchy sequence. Then there are numbers $l_{i} \in \mathbb{R}_{>0}$ and $\theta_{i} \in \mathbb{R}$ such that for all $i \in I$ we have $l_{x_{n}}\left(C_{i}\right) \rightarrow l_{i}$ and $\theta_{x_{n}}\left(C_{i}\right) \rightarrow \theta_{i}$ as $n \rightarrow \infty$.

Proof. By the definition of the length-spectrum distance, for every simple closed curve $\gamma$ on $S$, the sequence $\log \left(l_{x_{n}}(\gamma)\right)$ is a Cauchy sequence of real numbers. In particular, there exists a positive real number $l_{\gamma}$ such that $l_{x_{n}}(\gamma) \rightarrow l_{\gamma}$ as $n \rightarrow \infty$. Taking $\gamma=C_{i}$, we obtain $l_{x_{n}}\left(C_{i}\right) \rightarrow l_{C_{i}}=l_{i}$ as $n \rightarrow \infty$. Consider the curves $\beta_{i}, \beta_{i}^{\prime}$ as in Lemma 4.1, By using the formulae of [13] as in Lemma 4.1, we can see that $\left|\theta_{x_{n}}\left(C_{i}\right)\right|$ converges to a non-negative real number. If this number is zero, we put $\theta_{i}=0$; otherwise we choose $\theta_{i}$ such that $\left|\theta_{i}\right|$ is that number. To choose the sign of $\theta_{i}$ we use the limit of the sequence $\left|\theta_{x_{n}}\left(C_{i}\right)+2 \pi\right|$, the sign of $\theta_{i}$ being positive if this limit is greater that $l_{i}$; otherwise this sign is negative. With these choices we have $\theta_{x_{n}}\left(C_{i}\right) \rightarrow \theta_{i}$.

Lemma 4.3. Let $\left(x_{n}\right) \subset \mathcal{T}_{\text {ls }}\left(H_{0}\right)$ be a sequence, and let $x \in \mathcal{T}_{\text {ls }}\left(H_{0}\right)$ be such that $l_{x_{n}}\left(C_{i}\right) \rightarrow l_{x}\left(C_{i}\right)$ and $\theta_{x_{n}}\left(C_{i}\right) \rightarrow \theta_{x}\left(C_{i}\right)$. Then for every element $\gamma$ in $\mathcal{S}$, we have $l_{x_{n}}(\gamma) \rightarrow l_{x}(\gamma)$.

Proof. The closed curve $\gamma$ is compact; hence it is contained in a subsurface $S^{\prime}$ of $S$ that is the union of finitely many pairs of pants of the decomposition $\mathcal{P}$. Choose representatives in the equivalence classes of the structures $x_{n}$ and $x$ such that the boundary curves of $S^{\prime}$ are geodesics. Consider the restrictions $x_{n}^{\prime}$ and $x^{\prime}$ of our hyperbolic structures to $S^{\prime}$. On this finite type subsurface the Fenchel-Nielsen coordinates are finite-dimensional; hence the surfaces $x_{n}^{\prime}$ and $x^{\prime}$ are upper bounded in the sense of [2. That is, the length coordinates of $x$ and $x^{\prime}$ (with respect to a given pair of pants decomposition) are uniformly bounded from above. (This condition, used in 2 for surfaces of infinite type, is automatically satisfied for surfaces of finite type.) Since $d_{F N}\left(x_{n}^{\prime}, x^{\prime}\right) \rightarrow 0$, we obtain, from [2, Theorem 8.5], $d_{q c}\left(x_{n}^{\prime}, x^{\prime}\right) \rightarrow 0$, 
which, by Wolpert's Inequality, implies $d_{l s}\left(x_{n}^{\prime}, x^{\prime}\right) \rightarrow 0$. In particular, $l_{x_{n}}(\gamma) \rightarrow$ $l_{x}(\gamma)$.

Lemma 4.4. Let $\left(x_{n}\right) \subset\left(\mathcal{T}_{l s}\left(H_{0}\right), d_{l s}\right)$ be a Cauchy sequence, and let $x \in \mathcal{T}_{l s}\left(H_{0}\right)$ be such that $l_{x_{n}}\left(C_{i}\right) \rightarrow l_{x}\left(C_{i}\right)$ and $\theta_{x_{n}}\left(C_{i}\right) \rightarrow \theta_{x}\left(C_{i}\right)$. Then $d_{l s}\left(x_{n}, x\right) \rightarrow 0$.

Proof. By hypothesis, $\left(x_{n}\right)$ is a Cauchy sequence; that is,

$$
\forall \epsilon>0, \exists N: \forall n, m>N, d_{l s}\left(x_{n}, x_{m}\right)<\epsilon .
$$

Take an element $\gamma$ of $\mathcal{S}$. From the above property, we have, $\forall n, m>N$,

$$
\left|\log \frac{l_{x_{n}}(\gamma)}{l_{x_{m}}(\gamma)}\right|<\epsilon .
$$

By Lemma 4.3 we have $l_{x_{m}}(\gamma) \rightarrow l_{x}(\gamma)$; hence $\forall n>N$,

$$
\left|\log \frac{l_{x_{n}}(\gamma)}{l_{x}(\gamma)}\right| \leq \epsilon
$$

Taking the supremum over all $\gamma$ in $\mathcal{S}$, we have

$$
\forall \epsilon>0, \exists N: \forall n>N, d_{l s}\left(x_{n}, x\right) \leq \epsilon ;
$$

that is, $x_{n} \rightarrow x$.

Theorem 4.5. For any hyperbolic metric $H_{0}$ on $S$, the metric space $\left(\mathcal{T}_{l s}\left(H_{0}\right), d_{l s}\right)$ is complete.

Proof. This is a direct corollary of Lemmas 4.2 and 4.4. Take a Cauchy sequence $\left(x_{n}\right)$ in $\mathcal{T}_{l s}(X)$. By Lemma 4.2, we can find the limits of length and twist parameters $\left(l_{i}, \theta_{i}\right)$ of $C_{i}$. Use these numbers to construct a marked hyperbolic surface with Fenchel-Nielsen coordinates $\left(l_{i}, \theta_{i}\right)$. By Lemma 4.4 the sequence $x_{n}$ converges to this marked hyperbolic surface. Hence every Cauchy sequence has a limit.

Remarks 4.6. 1) Theorem 4.5 answers Question 2.22 of [10, which asks for a necessary and sufficient condition for a hyperbolic structure $H_{0}$ on a surface of infinite topological type under which the length-spectrum Teichmüller space $\left(\mathcal{T}_{l s}\left(H_{0}\right), d_{l s}\right)$ is complete.

2) The proof of Theorem 4.5 also works for surfaces of finite type. For such surfaces, the result was already known; see [10, Theorem 2.25].

Question 4.7. For every hyperbolic structure $H_{0}$ on an infinite type surface, we have the inclusion $\mathcal{T}_{q c}\left(H_{0}\right) \subset \mathcal{T}_{l s}\left(H_{0}\right)$, and we proved that this is not always an equality. Is it true that $\mathcal{T}_{q c}\left(H_{0}\right)$ is dense in $\mathcal{T}_{l s}\left(H_{0}\right)$ ? If this were true, $\left(\mathcal{T}_{l s}\left(H_{0}\right), d_{l s}\right)$ would be the metric completion of $\left(\mathcal{T}_{q c}\left(H_{0}\right), d_{l s}\right)$.

\section{MORE EXAMPLES}

In this section, we give examples of hyperbolic structures $H_{0}$ such that the restriction of the length-spectrum metric $d_{l s}$ to the Teichmüller space $\mathcal{T}_{q s}(H)$ is not complete. Of course, the hyperbolic structures do not satisfy Shiga's condition (1.4).

The first example is an adaptation of an example that was given in 9 . 
Example 5.1. Let $H_{0}$ be a hyperbolic surface with a pair of pants decomposition $\mathcal{P}=\left\{C_{i} \mid i \in I\right\}$ such that for some subsequence $C_{i_{k}}$ contained in the interior of $H_{0}, l_{H_{0}}\left(C_{i_{k}}\right)=\epsilon_{k}=e^{-k^{2}}$. For each $k=1,2, \ldots$, let $H_{k}$ be the hyperbolic surface obtained by a positive multi-Dehn twist of $H_{0}$ along $C_{i_{k}}$ of order $t_{k}=\left[\frac{\log \left|\log \epsilon_{k}\right|}{\epsilon_{k}}\right]$. Note that $\lim _{k \rightarrow \infty} t_{k}=\infty$ but $\lim _{k \rightarrow \infty} \frac{l_{H_{0}}\left(C_{i_{k}}\right) t_{k}}{\log \epsilon_{k}}=\lim _{k \rightarrow \infty} \frac{\log \left|\log \epsilon_{k}\right|}{\log \epsilon_{k}}=0$. We show that

$$
d_{l s}\left(H_{k}, H_{0}\right) \rightarrow 0, \text { while } d_{q c}\left(H_{k}, H_{0}\right) \rightarrow \infty .
$$

Let us first show that $d_{l s}\left(H_{k}, H_{0}\right) \rightarrow 0$. Recall that the length spectrum metric is defined by

$$
d_{l s}\left(H_{k}, H_{0}\right)=\max \left\{\log \sup _{\gamma} \frac{l_{H_{k}}(\gamma)}{l_{H_{0}}(\gamma)}, \log \sup _{\gamma} \frac{l_{H_{0}}(\gamma)}{l_{H_{k}}(\gamma)}\right\},
$$

where the supremum is taken over all essential simple closed curves $\gamma$ on $S$.

If for some $k$ a simple closed curve $\gamma$ does not intersect $C_{i_{k}}$, then the hyperbolic length of $\gamma$ is invariant under the twist along $C_{i_{k}}$. If $\gamma$ intersects $C_{i_{k}}$, we have

$$
l_{H_{k}}(\gamma)-i\left(\gamma, C_{i_{k}}\right) l_{H_{0}}\left(C_{i_{k}}\right) t_{k} \leq l_{H_{0}}(\gamma) \leq l_{H_{k}}(\gamma)+i\left(\gamma, C_{i_{k}}\right) l_{H_{0}}\left(C_{i_{k}}\right) t_{k} .
$$

As a result,

$$
d_{l s}\left(H_{k}, H_{0}\right)=\max \left\{\log \sup _{i\left(\gamma, C_{i_{k}}\right) \neq 0} \frac{l_{H_{k}}(\gamma)}{l_{H_{0}}(\gamma)}, \log \sup _{i\left(\gamma, C_{i_{k}}\right) \neq 0} \frac{l_{H_{0}}(\gamma)}{l_{H_{k}}(\gamma)}\right\} .
$$

We have

$$
\begin{aligned}
\log \frac{l_{H_{k}}(\gamma)}{l_{H_{0}}(\gamma)} & \leq \log \frac{l_{H_{0}}(\gamma)+i\left(\gamma, C_{i_{k}}\right) l_{H_{0}}\left(C_{i_{k}}\right) t_{k}}{l_{H_{0}}(\gamma)} \\
& =\log \left(1+\frac{i\left(\gamma, C_{i_{k}}\right) l_{H_{0}}\left(C_{i_{k}}\right) t_{k}}{l_{H_{0}}(\gamma)}\right) \\
& \leq \frac{i\left(\gamma, C_{i_{k}}\right) l_{H_{0}}\left(C_{i_{k}}\right) t_{k}}{l_{H_{0}}(\gamma)}
\end{aligned}
$$

and similarly,

$$
\log \frac{l_{H_{0}}(\gamma)}{l_{H_{k}}(\gamma)} \leq \log \frac{l_{H_{0}}(\gamma)}{l_{H_{0}}(\gamma)-i\left(\gamma, C_{i_{k}}\right) l_{H_{0}}\left(C_{i_{k}}\right) t_{k}} \leq \frac{i\left(\gamma, C_{i_{k}}\right) l_{H_{0}}\left(C_{i_{k}}\right) t_{k}}{l_{H_{0}}(\gamma)} .
$$

Thus, we have

$$
d_{l s}\left(H_{k}, H_{0}\right) \leq \sup _{i\left(\gamma, C_{i_{k}}\right) \neq 0} \frac{i\left(\gamma, C_{i_{k}}\right) l_{H_{0}}\left(C_{i_{k}}\right) t_{k}}{l_{H_{0}}(\gamma)} .
$$

We assume that every $\epsilon_{k}$ is less than some fixed constant $M>0$. Then there is a constant $C$ depending on $M$ such that $l_{H_{0}}(\gamma)$ is larger than $C i\left(\gamma, C_{i_{k}}\right)\left|\log \epsilon_{k}\right|$, as follows from the Collar Lemma [4. This lemma says that, for each simple closed geodesic with length $\ell$ less than $M$, there is a collar neighborhood of width larger than $w$, where $w$ is given by

$$
\sinh w=1 / \sinh (\ell / 2) .
$$

A simple computation shows that there is a constant $C$ depending on $M$ such that $w$ is larger than $C|\log \ell|$. Since any simple closed curve $\gamma$ which intersects $C_{i_{k}}$ should cross the collar neighborhood $i\left(\gamma, C_{i_{k}}\right)$ times, its hyperbolic length should be larger than $C i\left(\gamma, C_{i_{k}}\right)\left|\log \epsilon_{k}\right|$. As a result, the right hand side of inequality (5.1) tends to 0 as $k \rightarrow \infty$. Thus we have $d_{l s}\left(H_{k}, H_{0}\right) \rightarrow 0$. 
Example 5.2. Let $H_{0}$ be a hyperbolic surface with a hyperbolic pants decomposition $\mathcal{P}=\left\{C_{i}\right\}$ such that some subsequence of $C_{i_{k}}$ contained in the interior of $H_{0}$ satisfies the following:

(i) $l_{H_{0}}\left(C_{i_{k}}\right)=a_{k} \rightarrow \infty$.

(ii) For any geodesic arc $\alpha$ connecting two points (not necessary distinct) on $C_{i_{k}}$, but $\alpha \not \subset C_{i_{k}}, \alpha$ satisfies $l_{H_{0}}(\alpha)>k a_{k}$.

Let $H_{k}$ be the hyperbolic surface obtained by a positive Dehn twist of $H_{0}$ along $C_{i_{k}}$. Then for any simple closed curve $\gamma$ such that $i\left(C_{i_{k}}, \gamma\right) \neq 0, l_{H_{0}}(\gamma)$ is larger than $k i\left(\gamma, C_{i_{k}}\right) a_{k}$ and $l_{H_{0}}(\gamma)-i\left(\gamma, C_{i_{k}}\right) a_{k} \leq l_{H_{k}}(\gamma) \leq l_{H_{0}}(\gamma)-i\left(\gamma, C_{i_{k}}\right) a_{k}$. The arguments in Example 5.1 show that in the Teichmüller space $\mathcal{T}\left(H_{0}\right)_{q c}, d_{l s}\left(H_{k}, H_{0}\right) \rightarrow 0$ while $d_{q c}\left(H_{k}, H_{0}\right) \rightarrow \infty$.

Remark 5.3. Concrete examples satisfying conditions (i) and (ii) in Example 5.2 are constructed by Shiga [14] and Matsuzaki 12. Both examples satisfy the condition that given any constant $L$, there are finitely many simple closed geodesics on $H_{0}$ with hyperbolic length bounded above by $L$. Therefore these hyperbolic structures are different from those of Example 5.1. In the example of Shiga 14, the Riemann surface induced by $H_{0}$ is not complete. Matsuzaki [12] refined Shiga's construction to give a complete Riemann surface $H_{0}$ and then showed that for such an $H_{0}$, the Teichmüller modular group $\operatorname{Mod}\left(H_{0}\right)$ has only a countable number of elements.

Remark 5.4. The two above examples show that there exist hyperbolic surfaces $H_{0}$ of infinite topological type which do not satisfy Shiga's condition, such that $d_{q c}$ and $d_{l s}$ are not topologically equivalent on $\mathcal{T}_{q c}\left(H_{0}\right)$.

We conclude this paper with a new proof of a theorem due to Shiga [14].

Theorem 5.5. There exist surfaces $S$ of infinite topological type and hyperbolic structures on such surfaces such that the length-spectrum metric is not complete on $\left(\mathcal{T}_{q c}\left(H_{0}\right), d_{l s}\right)$.

Proof. We use the hyperbolic structures given in Examples 5.1] and 5.2. We shall prove that the structures in Example 5.1 satisfy the required property. The proof for the structures given in Example 5.2 is similar.

We consider the surface of Example [5.1, and we construct a Cauchy sequence in $\left(\mathcal{T}\left(H_{0}\right), d_{l s}\right)$ that does not have a limit.

Recall that $H_{0}$ is a hyperbolic surface with a pants decomposition $\mathcal{P}=\left\{C_{i}\right\}$ such that for some subsequence of $C_{i_{k}}$ contained in the interior of $H_{0}, l_{H_{0}}\left(C_{i_{k}}\right)=$ $\epsilon_{k}=e^{-k^{2}}$. We set $t_{k}=\left[\frac{\log \left|\log \epsilon_{k}\right|}{\epsilon_{k}}\right]$. Let $H_{1}$ be the hyperbolic surface obtained from $H_{0}$ by the positive multiple Dehn twist of order $t_{1}$ along $C_{i_{1}}$. More generally, for all $k \geq 1$, let $H_{k}$ be the hyperbolic surface obtained from $H_{k-1}$ by the positive multiple Dehn twist of order $t_{k}$ along $C_{i_{k}}$. Then as in the proof of Example 5.1, we can show that $d_{l s}\left(H_{m}, H_{n}\right) \rightarrow 0$ as $m, n \rightarrow \infty$. As a result, $\left(H_{k}\right)$ is a Cauchy sequence in $\left(\mathcal{T}\left(H_{0}\right), d_{l s}\right)$. We prove that $\left(H_{k}\right)$ has no limit in $\left(\mathcal{T}_{q c}\left(H_{0}\right), d_{l s}\right)$, by contradiction. Suppose there is a hyperbolic surface $H \in \mathcal{T}_{q c}\left(H_{0}\right)$ such that $d_{l s}\left(H_{k}, H\right) \rightarrow 0$. Consider the Fenchel-Nielsen coordinates determined by $H_{0}$ and $\mathcal{P}$. From the construction of the sequence $\left(H_{k}\right)$, the Fenchel-Nielsen coordinates of $H$ are $\left\{\left(l_{H}\left(C_{i}\right), \theta_{H}\left(C_{i}\right)\right)\right\}$, where $l_{H}\left(C_{i}\right)=l_{H_{0}}\left(C_{i}\right)$, with $\theta_{H}\left(C_{i_{k}}\right)-\theta_{H_{0}}\left(C_{i_{k}}\right)=2 \pi t_{k}$ and $\theta_{H}\left(C_{j}\right)-\theta_{H_{0}}\left(C_{j}\right)=0$ when $j \neq i_{k}$. We claim that $d_{q c}\left(H_{0}, H\right)=\infty$. As a result, $H$ does not belong to $\mathcal{T}_{q c}\left(H_{0}\right)$, which contradicts the assumption.

The fact that $d_{q c}\left(H_{0}, H\right)=\infty$ follows from Theorem 5.6 below, which says that if $d_{q c}\left(H_{0}, H\right)$ is finite, then $T_{k}$ is uniformly bounded. We recall that $T_{k}$ is 
a hyperbolic distance measured on the curve $C_{k}$, and therefore, in terms of the number of twists $t_{k}$, we have $T_{k}=t_{k} \times l_{H_{0}}\left(C_{k}\right)$; that is, $T_{k}=\left[\log \left|\log \epsilon_{k}\right|\right]$. Thus, if $d_{q c}\left(H_{0}, H\right)$ is finite, then $T_{k}$ is uniformly bounded, which contradicts the fact that $\left[\log \left|\log \epsilon_{k}\right|\right] \rightarrow \infty$ as $k \rightarrow \infty$.

The following result was used in the above proof. The statement is from [2, Theorem 7.6], adapted to our situation.

Theorem 5.6. Let $H_{0}$ be a hyperbolic surface with a hyperbolic pair of pants decomposition $P=\left\{C_{i}\right\}$, and assume that there exists a positive constant $L_{0}$ such that $l_{H_{0}}\left(C_{k}\right) \leq L$ for all $k=1,2, \ldots$ Let $C_{i_{k}}, k=1,2, \ldots$, be a subsequence of $\left(C_{i}\right)$, and let $T=\left(T_{k}\right), k=1,2, \ldots$, be a sequence of positive real numbers. Let $H_{t}$ be the hyperbolic metric obtained by a Fenchel-Nielsen twist along each geodesic curve $C_{i_{k}}$, of distance $T_{i}$ measured on $C_{i_{k}}$, for each $k$. Then if $d_{q c}\left(H_{0}, H_{t}\right)<M$, we have

$$
\sup _{k}\left|T_{k}\right| \leq C d_{q c}\left(H, H_{t}\right)
$$

where $C$ is a positive constant depending on $L$ and $M$.

Remark 5.7. Shiga's examples of hyperbolic structures are not complete (as metric spaces), whereas in our examples they are complete. To see this, note that since the geodesic length of each curve in the pairs of pants decomposition that we use is bounded uniformly from above, it follows that any closed ball of radius 1 on the surface is contained in a finite number of pairs of pants of the given decomposition, and therefore it is compact. Thus, by the theorem of Hopf-Rinow, the metric is complete (see [2, Lemma 4.7]).

\section{REFERENCES}

1. W. Abikoff, The real analytic theory of Teichmüller space, Lecture Notes in Mathematics, 820, Springer-Verlag (1980). MR590044 (82a:32028)

2. D. Alessandrini, L. Liu, A. Papadopoulos, W. Su, and Z. Sun, On Fenchel-Nielsen coordinates on Teichmüller spaces of surfaces of infinite type, to appear in Ann. Acad. Sci. Fenn. Math., arXiv:1003.0980

3. A. Basmajian, Hyperbolic structures for surfaces of infinite type. Trans. Amer. Math. Soc. 336, No. 1 (1993) 421-444. MR.1087051 (93e:30087)

4. P. Buser, Geometry and Spectra of Compact Riemann Surfaces, Birkhäuser (1992). MR 1183224 (93g:58149)

5. A. Douady and C. J. Earle, Conformally natural extensions of homeomorphisms of the circle, Acta Math. 157 (1986) 23-48. MR857678 (87j:30041)

6. A. Fathi, F. Laudenbach and V. Poénaru, Travaux de Thurston sur les surfaces, Astérisque 66-67 (1979). MR568308 (82m:57003)

7. Z. Li, Teichmüller metric and length spectrum of Riemann surfaces, Sci. Sinica (Ser. A) 29, (1986) 265-274. MR855233 (87k:32040)

8. L. Liu, On the length spectrums of non-compact Riemann surfaces, Ann. Acad. Sci. Fenn. Math. 24 (1999) 11-22. MR.1678001 (2001a:32020)

9. L. Liu, Z. Sun, and H. Wei, Topological equivalence of metrics in Teichmüller space. Ann. Acad. Sci. Fenn. Math. 33, No. 1 (2008) 159-170. MR.2386845 (2008k:32034)

10. L. Liu and A. Papadopoulos, Some metrics on Teichmüller spaces of surfaces of infinite type, to appear in the Transactions of the AMS.

11. S. Nag, The complex analytic theory of Teichmüller spaces, Canadian Mathematical Society Series of Monographs and Advanced Texts, John Wiley, 1988. MR.927291 (89f:32040)

12. K. Matsuzaki, A countable Teichmüller modular group, Trans. Amer. Math. Soc. 357, No. 8 (2004) 3119-3131. MR2135738 (2006f:30052)

13. T. Okai, Effects of a change of pants decompositions on their Fenchel-Nielsen coordinates, Kobe J. Math. 10 (1993) 215-223. MR.1270159 (95k:30094) 
14. H. Shiga, On a distance defined by length spectrum on Teichmüller space, Ann. Acad. Sci. Fenn. Math. 28 (2003) 315-326. MR 1996441 (2004i:30043)

15. W. P. Thurston, Three-Dimensional Geometry and Topology, Volume 1, Princeton University Press, Princeton, New Jersey, 1997. MR1435975 (97m:57016)

(Daniele Alessandrini) Institut de Recherche Mathématique Avancée, Université de Strasbourg and CNRS, 7 rue René Descartes, 67084 Strasbourg Cedex, France

E-mail address: daniele.alessandrini@gmail.com

Current address: Max-Plank-Institut für Mathematik, Vivatsgasse 7, D-53111 Bonn, Germany

(Lixin Liu) Department of Mathematics, Sun Yat-Sen (Zhongshan) University, 510275, Guangzhou, People's Republic of China

E-mail address: mcsllx@mail.sysu.edu.cn

(Athanase Papadopoulos) Institut de Recherche Mathématique Avancée, Université de Strasbourg and CNRS, 7 rue René Descartes, 67084 Strasbourg Cedex, France

E-mail address: athanase.papadopoulos@math.unistra.fr

(Weixu Su) Department of Mathematics, Sun Yat-Sen (Zhongshan) University, 510275, Guangzhou, People's Republic of China

E-mail address: su023411040@163.com 